Check for updates

Cite this: RSC Adv., 2017, 7, 47225

Received 8th August 2017

Accepted 2nd October 2017

DOI: $10.1039 / \mathrm{c} 7 \mathrm{ra0} 0866 \mathrm{~g}$

rsc.li/rsc-advances

\section{Rational design of hierarchical macroporous- mesoporous magnesium silicate for highly efficient removal of organic dye and $\mathrm{Pb}^{2+} \uparrow$}

\begin{abstract}
Chaoxia Zhao, (D) $\ddagger$ Jie Yang $\left(\mathbb{D} \ddagger^{*}\right.$ and Bo Jiang
A facile hydrothermal strategy has been developed to fabricate the hierarchical macroporous-mesoporous magnesium silicate (HMMgS) using macroporous siliceous foams (MOSF) as the silicon precursor and sacrificial template. The macroporous structure of HMMgS with a large surface area $\left(565 \mathrm{~m}^{2} \mathrm{~g}^{-1}\right.$ ) and high pore volume $\left(0.94 \mathrm{~cm}^{3} \mathrm{~g}^{-1}\right)$ is reproduced from the template MOSF, which can provide various active sites for the adsorption of pollutants. The mesopores stem from the stacking of magnesium silicate nanosheets on the scaffold of MOSF, facilitating the mass transportation of contaminants to the interfaces. Consequently, the usage efficiency of active sites on the surface can be greatly enhanced. Therefore, HMMgS exhibits the excellent adsorption capacities of $602 \mathrm{mg} \mathrm{g}^{-1}$ for methylene blue (MB) and $253 \mathrm{mg} \mathrm{g}^{-1}$ for $\mathrm{Pb}^{2+}$. Besides, HMMgS also demonstrates extraordinary recyclability with easy separation by sedimentation, showing its promising potential in practical wastewater treatment.
\end{abstract}

\section{Introduction}

With the fast development of modern society, large quantities of wastewater containing heavy metal ions and organic molecules have been discharged into the natural environment and caused severe health and environmental issues due to their high toxicity and nonbiodegradability. ${ }^{1-3}$ Various technologies have been applied to remove these contaminants from water, including chemical treatment, ${ }^{\mathbf{4}}$ photodegradation, ${ }^{5,6}$ membrane separation, ${ }^{7}$ adsorption, ${ }^{8}$ and so forth. ${ }^{9}$ Among these technologies, adsorption is considered to be a promising and effective way to remove various pollutants from wastewater due to its low price, high efficiency and easy operation. ${ }^{\mathbf{1 0 , 1 1}}$ As the contaminant standards in natural/drinking water become much stricter, the demand for low-cost and environmentally friendly adsorbents with high performance, easy separation and good recyclability becomes more urgent.

\footnotetext{
College of Chemistry, Sichuan University, Chengdu 610064, China. E-mail: j.yang@ scu.edu.cn; Fax: +86-28-85412291; Tel: +86-28-85418113

$\dagger$ Electronic supplementary information (ESI) available: Fig. S1: The TEM image and pore size distribution curve of MOSF; Fig. S2: Zeta potential of MB molecules as a function of $\mathrm{pH}$. Fig. $\mathrm{S} 3: \mathrm{Pb}^{2+}$ adsorption isotherms and kinetic isotherm of $\mathrm{Pb}^{2+}$; Fig. S4: Adsorption capacities of HMMgS for methyl orange (MO), acid blue 83 (AB83), methyl blue (MLB) and $\mathrm{Cr}(\mathrm{vr})$. Table S1: MB adsorption properties of MOSF, HMMgS and related adsorbents reported in literatures; Table S2: The equilibrium times of adsorbents for the removal of MB and $\mathrm{Pb}^{2+}$ reported in literatures; Table S3: Adsorption parameters of Langmuir model and pseudo-second-order adsorption kinetic constants for $\mathrm{Pb}^{2+}$ adsorption. See DOI: 10.1039/c7ra08766g

\$ These authors contribute equally to this paper.
}

Up to now, numerous adsorbents with good performance have been development for the removal of organic dyes and heavy metal ions from wastewater, such as porous carbon, ${ }^{\mathbf{1 2}}$ natural zeolites ${ }^{13}$ metal oxides, ${ }^{14}$ and silicates. ${ }^{15,16}$ Among them, magnesium silicates have attracted much attention due to their abundance, stable and environmentally friendly features as well as the fascinating structures. ${ }^{\mathbf{1 0 , 1 7 - 2 0}}$ Large interfaces and appropriate pore structure are the two of the most important criteria for a good adsorbent, ${ }^{21,22}$ since large surface areas could produce more adsorption sites and suitable pore structure may accelerate the rate of mass transportation of pollutants. ${ }^{23}$ Consequently, previous studies on magnesium silicate for the removal of pollutants mainly focus on the fabrication of silicates with various nanostructures and morphologies, such as hollow sphere, ${ }^{10,24}$ hollow nanofiber/nanotube, ${ }^{11,22,25}$ core-shell and hierarchical structures, ${ }^{\mathbf{1 6 , 1 9 , 2 3 , 2 6 - 2 9}}$ to create large interfaces for enhancing the removal performance. However, the importance of the pore size distribution on the adsorption performance is underestimated in most of the cases. Compared to the effect of interfaces, the pore size distribution normally exhibits more significant influence on the adsorption performance. ${ }^{\mathbf{3 0 , 3 1}}$ Therefore, some magnesium silicate materials with hierarchical porous nanostructures ${ }^{\mathbf{1 6 , 2 8}}$ have been made as the adsorbents due to the importance of pore structures. However, their hierarchical pores are normally in the mesoporous range (below $50 \mathrm{~nm}$ ), in which the mass transportation and diffusion of pollutants may still be hindered to some extent. Due to the nanostructures with inappropriate pore size distribution, which may cause the obstacle for the mass transportation of contaminants and consequent low usage efficiency of active sites on interfaces, most of the magnesium silicate materials 
with various nanostructures still exhibit the unsatisfactory adsorption performance for organic dyes and/or heavy metal ions even owning large interfaces. Therefore, it is necessary to design a magnesium silicate based adsorbent with both large interfaces and properly distributed pore size to enhance the adsorption performance.

Herein, we reported a facile route to fabricate hierarchical macroporous-mesoporous magnesium silicate (HMMgS) for highly efficient removal of cationic organic dye methylene blue (MB) and heavy metal ion $\mathrm{Pb}^{2+}$ through the mild hydrothermal reaction. As illustrated in Scheme 1, macroporous siliceous foams (MOSF) (pore size $=\sim 100 \mathrm{~nm}$ ), which are prepared through a supra-assembly approach using non-ionic block copolymers as supramolecular templates, ${ }^{32,33}$ was used as both sacrificial template and silicon source. In the basic hydrothermal condition, silica template MOSF can be gradually dissolved to produce silicate ions, which then were released from the template and reacted with magnesium ions in the solution to form magnesium silicate nanoparticles around the scaffold of MOSF. Magnesium silicate nanoparticles grew into nanosheets and further self-assembled on the scaffold of MOSF, accompanied by the gradual dissolution of MOSF. After the complete consumption of silica template, magnesium silicate with the macroporous structure reproduced from the template can be obtained. Besides, the stacking of magnesium silicate nanosheets on the scaffold of MOSF can also create quantities of mesopores. Therefore, using MOSF as the template, magnesium silicate with hierarchical nanostructure composed of macropores and mesopores can be successfully prepared

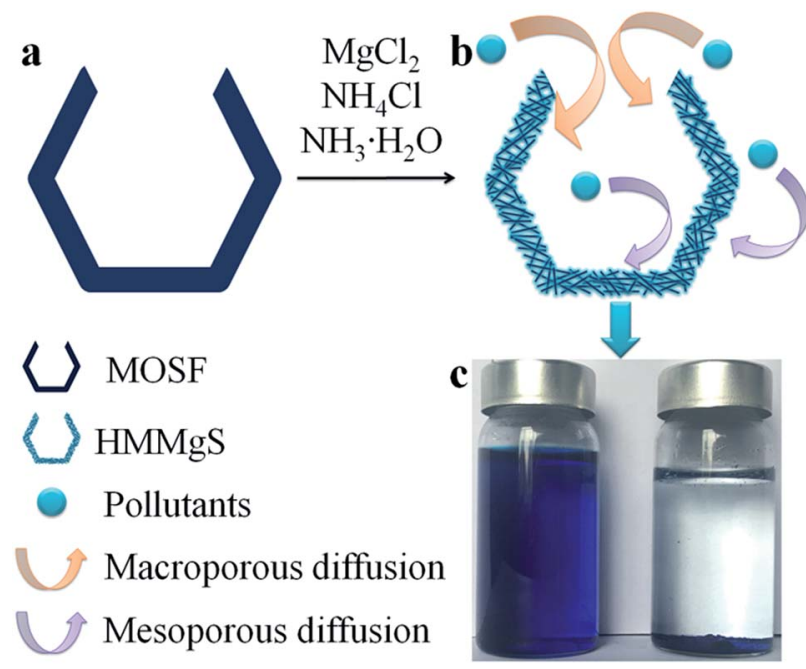

Scheme 1 Schematic illustration of the preparation of HMMgS and the adsorption of pollutants by HMMgS. Under basic hydrothermal condition, MOSF (a) can be dissolved into silicate ions, which further reacted with magnesium ions to form magnesium silicate nanoparticles. Magnesium silicate nanoparticles grew into nanosheets and self-assemble on the template of MOSF to form HMMgS (b) composed of macropores and mesopores. Pollutants can diffuse into the macropores and mesopores freely and further be adsorbed by the active sites on the surface of HMMgS. After adsorption, HMMgS can be easily separated by sedimentation (c). The pore size of MOSF and HMMgS, and the size of pollutants are not to scale. through the facile hydrothermal strategy. The large surface area $\left(565 \mathrm{~m}^{2} \mathrm{~g}^{-1}\right)$ and high pore volume $\left(0.94 \mathrm{~cm}^{3} \mathrm{~g}^{-1}\right)$ of $\mathrm{HMMgS}$ provides numerous active sites for the adsorption of pollutants. In addition, the hierarchical pore structure consisting of both marcopores and mesopores favours the mass transportation of contaminants to the interfaces, so the usage efficiency of active sites on the surfaces can be greatly enhanced. Hence, HMMgS exhibits the excellent adsorption capacities of $602 \mathrm{mg} \mathrm{g}^{-1}$ for

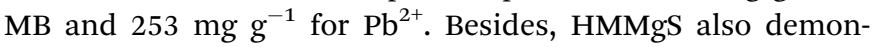
strates the extraordinary recyclability with the easy separation by sedimentation, showing its promising potential in the practical wastewater treatment.

\section{Experimental}

\section{Materials}

All the chemicals were used as received without further purification. $\mathrm{EO}_{20} \mathrm{PO}_{70} \mathrm{EO}_{20}$ [denoted as $\mathrm{P} 123$, where $\mathrm{EO}$ is poly(ethylene oxide) and $\mathrm{PO}$ is poly-(propylene oxide)] was purchased from Sigma. Tetramethyl orthosilicate (TMOS) was purchased from Shanghai Energy Chemical Co. Ltd, China. Other chemicals were purchased from Chengdu Kelong Chemical Co. Ltd, China.

\section{Preparation of MOSF}

MOSF materials were prepared using P123 as the soft template according to the method reported previously. ${ }^{34}$ Briefly, P123 $(1 \mathrm{~g})$ and $\mathrm{Na}_{2} \mathrm{SO}_{4}(1.7 \mathrm{~g}, 0.40 \mathrm{M})$ were dissolved in $\mathrm{pH}=5.0$ NaAc-HAc (Ac $=$ acetate) buffer solution $(30 \mathrm{~g})\left(C_{t}=0.02 \mathrm{M}\right.$, where $\left.C_{t}=C_{\mathrm{NaAc}}+C_{\mathrm{HAc}}\right)$ at $35^{\circ} \mathrm{C}$, then a homogeneous solution can be obtained under stirring. To this solution mixture, TMOS $(1.52 \mathrm{~g})$ was added. After stirring for 5 minutes, the mixture was kept in a static condition for $24 \mathrm{~h}$ and then hydrothermally treated at $100{ }^{\circ} \mathrm{C}$ for another $24 \mathrm{~h}$. The white precipitates were filtered, repeatedly washed with water to remove the inorganic salts, and then dried at room temperature. The final MOSF products were obtained by calcination at $550{ }^{\circ} \mathrm{C}$ for $5 \mathrm{~h}$.

\section{Preparation of hierarchical macroporous-mesoporous metal silicates}

At room temperature, magnesium nitrate $(0.75 \mathrm{mmol})$ and ammonia chloride (10 mmol) were dissolved in deionized water $(30 \mathrm{~mL})$, and then ammonia solution $(1 \mathrm{~mL}, 28 \%)$ was added to obtained the solution A. MOSF ( $0.1 \mathrm{~g}$ ) was homogeneously dispersed in deionized water $(20 \mathrm{~mL})$ to get the solution $\mathrm{B}$. Solution A and B were mixed. After stirring for $10 \mathrm{~min}$, the mixture was transferred into a Teflon autoclave $(100 \mathrm{~mL})$ and hydrothermally treated in a heating oven $\left(14 \mathrm{~h}\right.$ at $\left.140{ }^{\circ} \mathrm{C}\right)$. After cooling to room temperature, the precipitate was filtered, repeatedly washed with water and ethanol, and then dried in a $60{ }^{\circ} \mathrm{C}$ oven overnight.

\section{Adsorption experiments}

Methylene blue (MB) adsorption isotherms were acquired through batch experiments. The as-prepared HMMgS (0.02 g) was mixed with solution containing different concentration of 
MB (ranging from 100 to $\left.600 \mathrm{mg} \mathrm{L}^{-1}\right)(40 \mathrm{~mL})$, followed by shaking $(200 \mathrm{rpm})$ at $25{ }^{\circ} \mathrm{C}$ for $24 \mathrm{~h}$ to achieve adsorption equilibrium. After centrifugation, the MB concentration was analysed by UV-Vis absorption spectroscopy at a wavelength of 664 nm (Mapada, UV-3100PC, China).

All the adsorption data were fitted with the Langmuir, Freundlich, and Dubinin-Radushkevitch isotherm models. The Langmuir isotherm is expressed as follow:

$$
q_{\mathrm{eq}}=\frac{b Q_{\mathrm{max}} C_{\mathrm{eq}}}{1+b C_{\mathrm{eq}}}
$$

where $Q_{\max }$ is the maximum adsorption amount of $\mathrm{MB}\left(\mathrm{mg} \mathrm{g}^{-1}\right)$, $C_{\mathrm{eq}}$ is the equilibrium solute concentration $\left(\mathrm{mg} \mathrm{L}^{-1}\right), b$ is Langmuir constant $\left(\mathrm{L} \mathrm{mg}^{-1}\right)$ which relates to the adsorption affinity, $q_{\mathrm{eq}}$ is the amount adsorbed at equilibrium $\left(\mathrm{mg} \mathrm{g}^{-1}\right)$. According to the value of $b$, the separation factor or equilibrium factor $R_{\mathrm{L}}$ can be calculated using the following equation: ${ }^{35}$

$$
R_{\mathrm{L}}=\frac{1}{1+b C_{0}}
$$

where $C_{0}$ represents the initial concentration of $\mathrm{MB}\left(\mathrm{mg} \mathrm{g}^{-1}\right) \cdot R_{\mathrm{L}}$ in the range of $0<R_{\mathrm{L}}<1$ and $R_{\mathrm{L}}>1$ indicates favourable adsorption and unfavourable adsorption, ${ }^{36,37}$ respectively. $R_{\mathrm{L}}=$ 1 stands for the linear adsorption and $R_{\mathrm{L}}=0$ represents the irreversible adsorption.

The Freundlich isotherm is expressed as follow:

$$
q_{\mathrm{eq}}=K C_{\mathrm{eq}}^{1 / n}
$$

where $K\left(\mathrm{mg}^{(1-n)} \mathrm{L}^{n} \mathrm{~g}^{-1}\right)$ is the Freundlich constant that relates to the adsorption capacity, and $n$ is the index that stands for adsorption strength.

Dubinin-Radushkevitch isotherm model based on Polanyi theory was utilized to express the MB adsorption mechanism of HMMgS, which can be expressed as:

$$
\text { In } q_{\mathrm{eq}}=\text { In } Q_{\max }-K \varepsilon^{2}
$$

where $q_{\text {eq }}$ is the amount adsorbed at equilibrium $\left(\mathrm{mg} \mathrm{g}^{-1}\right), Q_{\max }$ is the theoretical monolayer adsorption capacity of $\mathrm{MB}$ $\left(\mathrm{mg} \mathrm{g}^{-1}\right), K$ is the constant representing the mean free energy of adsorption $\left(\mathrm{mol}^{2} \mathrm{~kJ}^{-2}\right), \varepsilon$ is the Polanyi potential $\left(\mathrm{kJ} \mathrm{mol}^{-1}\right)$. $\varepsilon=R T \ln \left[1+\left(1 / C_{\mathrm{eq}}\right)\right]$, in which $C_{\mathrm{eq}}$ is the equilibrium solute concentration $\left(\mathrm{mg} \mathrm{L}^{-1}\right), R$ is the gas constant $\left(\mathrm{J} \mathrm{mol}^{-1} \mathrm{~K}^{-1}\right)$, and $T$ is the temperature (K).

The adsorption kinetic experiments were carried out by mixing MOSF or HMMgS (0.02 g) with $40 \mathrm{~mL}$ of solution with an initial $\mathrm{MB}$ concentration of $100 \mathrm{mg} \mathrm{L}^{-1}$. The contact time $(t)$ is calculated after the addition of adsorbent into the solution. The suspension was placed in a rotary shaker with the speed of $200 \mathrm{rpm}$ at $25{ }^{\circ} \mathrm{C}$ for $24 \mathrm{~h}$. The suspension was separated by centrifugation at controlled $t$ during the $24 \mathrm{~h}$ experiment for analyses.

The kinetic data of HMMgS were fitted to the pseudo firstorder kinetic model, pseudo second-order kinetic model, and intraparticle diffusion model.

The pseudo first-order kinetic model can be represented in the following form:

$$
\frac{\mathrm{d} q_{t}}{\mathrm{~d} t}=k_{1}\left(q_{\mathrm{eq}}-q_{t}\right)
$$

The pseudo second-order kinetic model can be expressed as:

$$
\frac{\mathrm{d} q_{t}}{\mathrm{~d} t}=k_{2}\left(q_{\mathrm{eq}}-q_{t}\right)^{2}
$$

The Weber-Morris intraparticle diffusion equation is written as: $^{38}$

$$
q_{t}=k_{\mathrm{dif}} \sqrt{t}+c
$$

where $q_{\text {eq }}$ is the sorption capacity at equilibrium $\left(\mathrm{mg} \mathrm{g}^{-1}\right), q_{t}$ is the amount adsorbed at a contact time $t\left(\mathrm{mg} \mathrm{g}^{-1}\right), k_{1}\left(\mathrm{~min}^{-1}\right), k_{2}$ $\left(\mathrm{g} \mathrm{mg}^{-1} \mathrm{~min}^{-1}\right)$ and $k_{\text {dif }}\left(\mathrm{mg} \mathrm{g}^{-1} \mathrm{~min}^{-0.5}\right)$ are the rate constants of the pseudo first order kinetic model, pseudo second order kinetic model, and intraparticle diffusion model, respectively. $C$ is the constant $\left(\mathrm{mg} \mathrm{g}^{-1}\right)$. Nonlinear least-squares regression analysis was applied to acquire the best estimation of all constants for all the models in MB adsorption test.

The effect of ionic strength were studied by adding $40 \mathrm{mg}$ of HMMgS into $20 \mathrm{~mL}$ of $600 \mathrm{mg} \mathrm{L}^{-1} \mathrm{MB}$ solution with different concentration of $\mathrm{NaCl}\left(0.05,0.1,0.2,0.3\right.$ and $\left.0.4 \mathrm{~mol} \mathrm{~L}^{-1}\right)$ without adjusting $\mathrm{pH}$. After shaking in a rotary shaker with the speed of $200 \mathrm{rpm}$ at $25{ }^{\circ} \mathrm{C}$ for $24 \mathrm{~h}$, the suspension was separated by centrifugation for analyses.

$\mathrm{Pb}^{2+}$ adsorption experiments were performed in a similar way as $\mathrm{MB}$ adsorption. The concentration of $\mathrm{Pb}^{2+}$ was analysed by inductively coupled plasma atomic emission spectroscopy (ICP-AES, IRIS Adv).

\section{Characterisation}

Synthesised materials were comprehensively characterised using X-ray diffraction (XRD, Bruker D8 Advanced X-ray Diffractometer with Ni-filtered $\mathrm{Cu} \mathrm{K} \alpha$ radiation at a voltage of $40 \mathrm{mV}$ and a current of $30 \mathrm{~mA}$ ), scanning electron microscopy (SEM, Philips XL300 operated at $20 \mathrm{kV}$ and JEOL JSM-6460 equipped with energy-dispersive spectroscopy (EDS)), and transmission electron microscopy (TEM, JEOL 2100 operated at $200 \mathrm{kV}$ ). The samples for TEM measurements were dispersed in ethanol by ultrasonication for $5 \mathrm{~min}$ and then supported onto a holey carbon film on a copper grid. The $\mathrm{N}_{2}$ adsorptiondesorption isotherms were measured at $-196{ }^{\circ} \mathrm{C}$ on a nitrogen adsorption apparatus (Quadrasorb SI, Quantachrome) after degassing the samples at $180{ }^{\circ} \mathrm{C}$ for 6 hours. The BrunauerEmmett-Teller (BET) surface areas were determined from the adsorption branch of the isotherm in a relative pressure ranging from 0.05 to 0.30 . The pore size distribution (PSD) was determined using Broekhoff and de Boer (BdB) model and Barrett, Joyner and Halenda $(\mathrm{BJH})$ method from the adsorption branch. The $\mathrm{pH}$ of the solution was measured by TPS labCHEM-pH meter. X-ray photoelectron spectroscopy (XPS) measurements were performed with a Kratos Axis Ultra X-ray photoelectron spectrometer (Perkin-Elmer). All spectra were acquired at a basic pressure of $2 \times 10^{-7}$ Torr with $\mathrm{Mg} \mathrm{K} \alpha$ excitation at $15 \mathrm{kV}$. All the results were analysed using the CasaXPS software and 
corrected by referencing the $\mathrm{C} 1 \mathrm{~s}$ peak at $284.8 \mathrm{eV} \cdot{ }^{39}$ Fourier transform infrared (FTIR) spectra were collected on a Bruker Tensor 27 FTIR spectrometer using $\mathrm{KBr}$ pellets. For each spectrum, 64 scans were collected at a resolution of $4 \mathrm{~cm}^{-1}$ over the range $400-4000 \mathrm{~cm}^{-1}$.

\section{Results and discussion}

The morphologies of MOSF and HMMgS were first characterised by scanning electron microscopy (SEM) and transmission electron microscopy (TEM). The typical foam-like macroporous structure of MOSF with the pore size of about $100 \mathrm{~nm}$ and the silica wall thickness of $\sim 7 \mathrm{~nm}$ can be clearly identified from SEM and TEM images (Fig. 1a, inset of Fig. 1a, and $\mathrm{S} 1 \mathrm{a} \dagger)$. MOSF is an appropriate sacrificial template for the synthesis of macroporous magnesium silicate material due to its large pore size and open pore structure. Because of such unique pore structure, magnesium ions can diffuse into the pores of MOSF freely and distribute around the silica wall homogeneously. During the hydrothermal treatment in basic condition, the thin silica wall can be easily dissolved and react with magnesium ions, and then magnesium silicate can be formed in situ. ${ }^{22}$ After reacting with magnesium ions, MOSF acting as the silica source can be converted to HMMgS. HMMgS has the aggregated spherical morphology (Fig. 1b). The magnified SEM image demonstrates that the surface of HMMgS becomes rough after the hydrothermal treatment (Fig. 1c). Besides, HMMgS also shows the macroporous structure which is assembled by quantities of thin nanosheets with the thickness of $\sim 13 \mathrm{~nm}$ (inset of Fig. 1c). ${ }^{11}$ The structure of HMMgS was further explored by TEM. Under TEM observation, the macropores of HMMgS can be clearly discerned (Fig. 1d), which originate from the macropores of sacrificial template MOSF. It is worth noting that very few nanosheets can be observed in the TEM image. Instead, a great number of needle-like structures with the diameter of $\sim 15 \mathrm{~nm}$ are found in the TEM images of HMMgS (indicated by black arrows in Fig. 1d and e), which cannot match the results of SEM. However, these nanoneedles in the TEM images are nanosheets in the SEM image actually. When a nanosheet is parallel to the beam direction, its projection in the TEM image shows a needle-like structure, whose diameter represents the thickness of the nanosheet. Only viewed perpendicular to the beam direction, the nano-scale lamellae can be identified. According to this explanation, it can be concluded that the macropores of HMMgS are formed through the self-assembly of magnesium silicate nanosheets with the thickness of $\sim 15 \mathrm{~nm}$, which is in accordance with SEM results. Apart from the macropores, the stacking of nanosheets can also lead to the formation of mesopores, which is confirmed by the following nitrogen adsorption analysis. The energydispersive spectroscopy (EDS) confirms the presence of $\mathrm{Si}, \mathrm{O}$ and $\mathrm{Mg}$ (Fig. 1f), indicating the formation of magnesium silicate. HMMgS with a hierarchical porous structure consisting of macropores and mesopores may favour the fast transportation and diffusion of pollutants, which makes it a promising adsorbent for the wastewater treatment.
To confirm the successful preparation of HMMgS using MOSF as the sacrificial template through the mild hydrothermal treatment strategy, HMMgS was characterised by the wide-angle X-ray diffraction (XRD). Fig. 2a displays the XRD patterns of MOSF and HMMgS. Compared with the XRD pattern of MOSF showing a broad diffraction peak centred at $2 \theta=\sim 22^{\circ}$ due to its composition of amorphous silica, ${ }^{40}$ the pattern of HMMgS exhibits five well-defined peaks at $2 \theta=19.7^{\circ}, 34.7^{\circ}$, $53.4^{\circ}, 60.7^{\circ}$ and $72.1^{\circ}$. These diffractions can be assigned to the (020), (200), (-241), (-332) and (-264) reflections of magnesium silicate $\left(3 \mathrm{MgO} \cdot 4 \mathrm{SiO}_{2} \cdot 2 \mathrm{H}_{2} \mathrm{O}\right.$, JCPDS no. 03-0174), respectively, indicating the transformation from $\mathrm{SiO}_{2}$ to magnesium silicate. ${ }^{10}$ The broad diffractions of HMMgS also suggest the crystals of HMMgS with the imperfect degree of crystallinity are in the nano-scale range.

The successfully preparation of HMMgS was further confirmed by Fourier transform infrared (FTIR) analysis (Fig. 2b). The spectrum of MOSF demonstrates two peaks at 802 and $1085 \mathrm{~cm}^{-1}$, which can be indexed to the symmetric and asymmetric Si-O-Si stretching. ${ }^{41}$ Adsorption bands at 1631 and $3431 \mathrm{~cm}^{-1}$ can be contributed to the stretching and bending modes of physiosorbed water in the sample. Compared with MOSF, HMMgS shows three new bands at 667, 1028 and $3680 \mathrm{~cm}^{-1}$, suggesting the formation of magnesium silicate. ${ }^{23,42}$ The formation of magnesium silicate was also supported by the XPS analysis. The survey spectrum of HMMgS (Fig. 2c) demonstrates the presence of $\mathrm{Mg}, \mathrm{Si}$ and $\mathrm{O}$, in according with the EDS analysis. The photoelectron peak located at $102.6 \mathrm{eV}$ is attributed to Si 2p of magnesium silicate (inset of Fig. 2c), ${ }^{22}$ considering the characteristic peak of $\mathrm{Si} 2 \mathrm{p}$ for $\mathrm{SiO}_{2}$ is around $103.8 \mathrm{eV} .^{43}$ The above results confirm the magnesium silicate with hierarchical macroporous-mesoporous nanostructure can be successfully fabricated by the facile hydrothermal treatment method using MOSF as the sacrificial template.

The pore structures and structural parameters of MOSF and HMMgS were analysed by nitrogen sorption measurements. Sacrificial template MOSF demonstrates a characteristic type II adsorption isotherm with relatively large hysteresis loop and the capillary condensation step at the relative pressure larger than 0.9 (Fig. 2d), confirming the presence of macropores. ${ }^{32}$ Similarly, HMMgS also shows a typical type II adsorption isotherm with $\mathrm{H}_{3}$ hysteresis loop (Fig. 2d), indicating the macroporous structure of HMMgS is composed of platelike particles. ${ }^{\mathbf{4}}$ Compared with MOSF with the surface area of $265 \mathrm{~m}^{2} \mathrm{~g}^{-1}$ and pore volume of $1.26 \mathrm{~cm}^{3} \mathrm{~g}^{-1}$, the increase to $565 \mathrm{~m}^{2} \mathrm{~g}^{-1}$ in the surface area and the reduction to $0.94 \mathrm{~cm}^{3} \mathrm{~g}^{-1}$ in the pore volume of HMMgS can be identified. It is worth noting that HMMgS has a characteristic hierarchical porous nanostructure consisting of macropores and mesopores. The macropores in the range of 140-190 $\mathrm{nm}$ according to the pore size distribution curve calculated from the adsorption branch using Broekhoffde Boer (BdB) model ${ }^{45}$ (Fig. 2e) originates from the pores of template MOSF (98 nm, Fig. S1 $\mathrm{b}^{\dagger}$ ), and the mesopores with the size distribution centred at $5.8 \mathrm{~nm}$ (calculated by Barrett, Joyner and Halenda (BJH) method from the adsorption branch) is generated from the loose assembly of platelike particles (Fig. 2f). The high surface area, hierarchical porous 

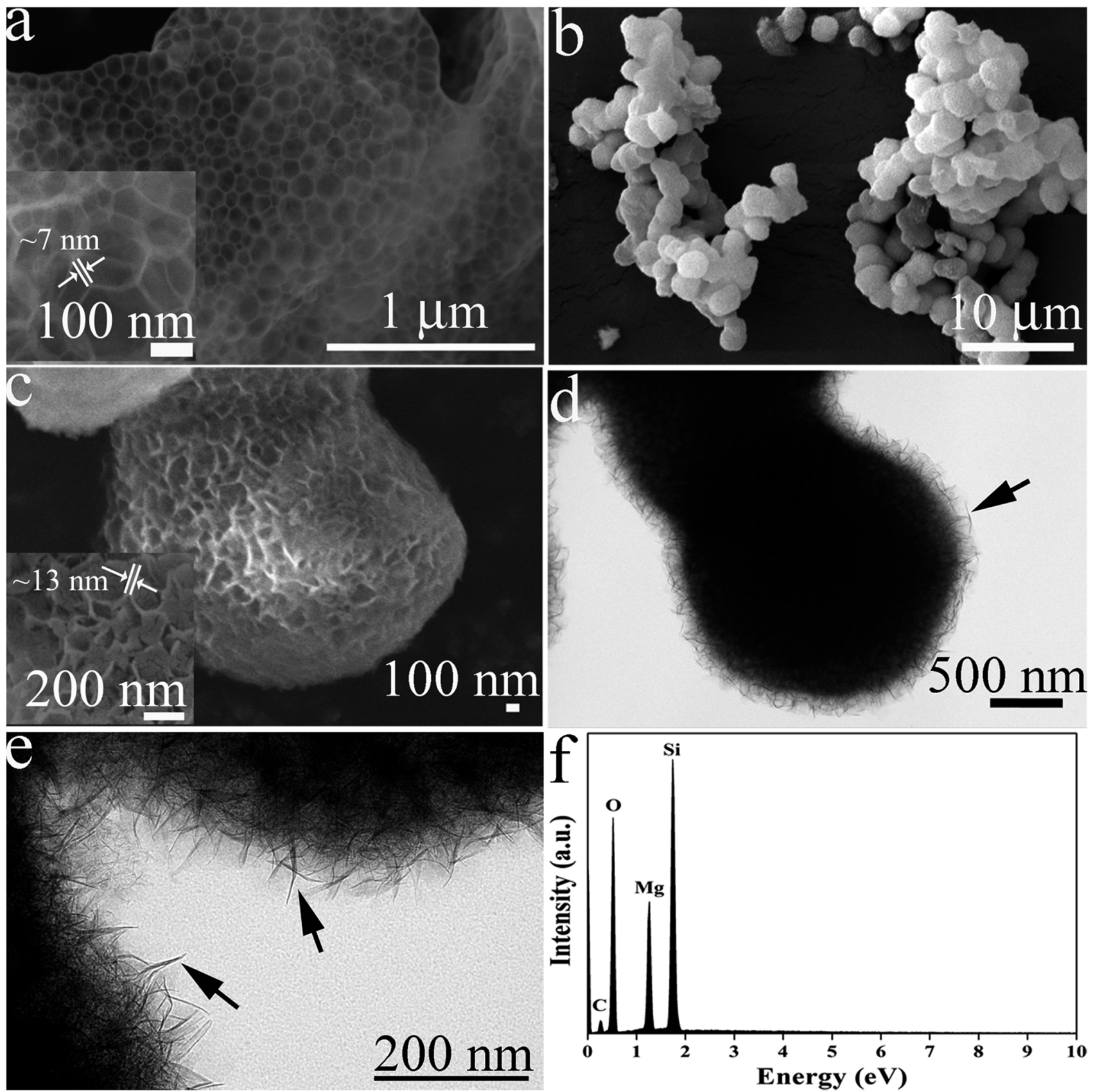

Fig. 1 (a) The SEM image of MOSF; (b) SEM and (c) magnified SEM images of HMMgS; (d) TEM and (e) magnified TEM images of HMMgS; (f) EDS spectrum of HMMgS. Insets of (a) and (c) are magnified SEM images of MOSF and HMMgS, respectively.

nanostructure and high pore volume make HMMgS to be a highly efficient adsorbent for water quality control and protection.

Magnesium silicate with negatively charged surface could exhibit extraordinary adsorption performance for cationic dyes. ${ }^{10} \mathrm{MB}$ is a common cationic dye in the textile industry and here it was selected as a model organic pollutant. To evaluate the MB adsorption capacity of HMMgS, the adsorption experiments were carried out. Both Langmuir and Freundlich isotherms were employed to analyse the adsorption data (Fig. 3a), and the fitting results are listed in Table 1. Although both Langmuir and Freundlich models are suitable for the fitting of adsorption isotherms, Langmuir model shows a better fit according to its higher correlation coefficient $\left(R^{2}\right.$, Table 1$)$, suggesting the adsorption of $\mathrm{MB}$ on HMMgS occurs on the homogeneous surface through electrostatic interaction..$^{10,18}$ The pristine MOSF can adsorb little MB with the adsorption capacity of $115 \mathrm{mg} \mathrm{g}^{-1}$. In comparison, HMMgS demonstrates the enhanced adsorption capacity of MB. The maximum MB adsorption capacity according to the fitting result of Langmuir model is $602 \mathrm{mg} \mathrm{g}^{-1}$, almost 5-6 times higher than that of pristine MOSF and also higher than most of the previously 

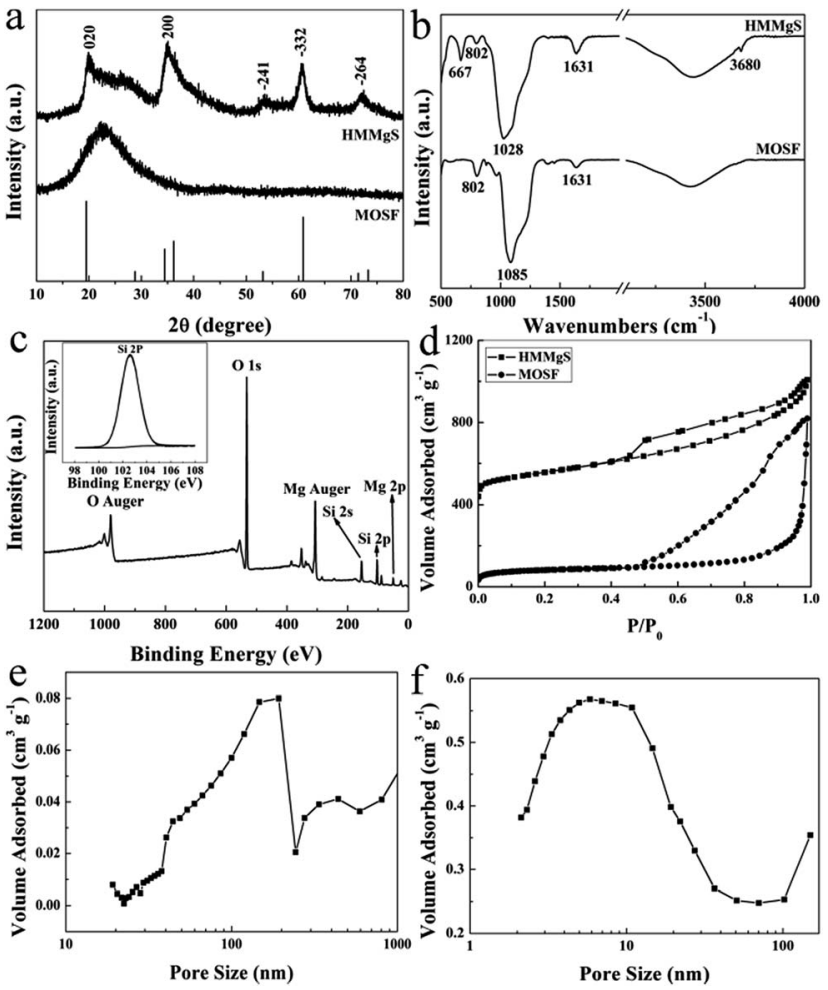

Fig. 2 (a) The wide-angle XRD patterns and (b) FT-IR spectra of HMMgS and MOSF; (c) XPS survey scan of HMMgS (inset: the corresponding fine spectrum of $\mathrm{Si} 2 \mathrm{p}$ ); (d) the nitrogen adsorptiondesorption isotherms of HMMgS and MOSF; (e) pore size distribution curves calculated by Broekhoff-de Boer model; and (f) pore size distribution curves calculated by Barrett, Joyner and Halenda (BJH) method from the adsorption branch.
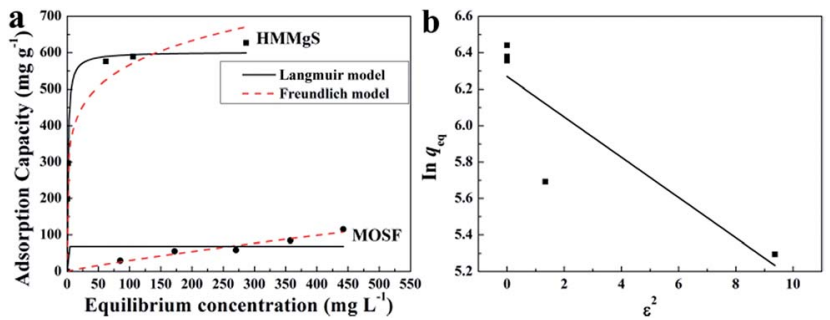

Fig. 3 (a) MB adsorption isotherms of HMMgS and MOSF; (b) DubininRadushkevich plot for the adsorption of MB by HMMgS.

reported MB adsorbents (Table S1†), indicating the high MB removal efficiency of HMMgS. According to Langmuir isotherm model, the surface of HMMgS can adsorb only one layer of MB molecule through electrostatic interaction. The theoretical monolayer adsorption capacity of $\mathrm{MB}$ can be calculated by the equation $S \times M_{\text {dye }} \times 1000 / S_{\text {dye }} \times N$, where $S$ is the surface area of HMMgS, $M_{\text {dye }}$ is the molecular weight of MB, $S_{\text {dye }}$ is the projection area of one MB molecule, and $N$ is the Avogadro constant. ${ }^{46}$ According to this equation, $1 \mathrm{~g}$ of $\mathrm{HMMgS}$ can adsorb $545 \mathrm{mg}$ of MB in maximum, which is lower than the experimental result of $602 \mathrm{mg}$. The difference between theoretical calculation and experiment results can be explained by
Table 1 Adsorption parameters of Langmuir, Freundlich model and Dubinin-Radushkevich model

\begin{tabular}{|c|c|c|c|}
\hline \multirow[b]{2}{*}{ Sample } & \multicolumn{3}{|l|}{ Langmuir model } \\
\hline & $Q_{\max }\left(\mathrm{mg} \mathrm{g}^{-1}\right)$ & $b\left(\mathrm{~L} \mathrm{mg}^{-1}\right)$ & $R^{2}$ \\
\hline MOSF & 68.9 & $1.830 \times 10-45$ & 0.357 \\
\hline \multirow[t]{2}{*}{ HMMgS } & 602 & 0.759 & 0.978 \\
\hline & \multicolumn{3}{|c|}{ Freundlich model } \\
\hline Sample & $\begin{array}{l}K \\
\left(\mathrm{mg}^{(1-n)} \mathrm{L}^{n} \mathrm{~g}^{-1}\right)\end{array}$ & $n$ & $R^{2}$ \\
\hline MOSF & 0.590 & 1.17 & 0.955 \\
\hline \multirow[t]{2}{*}{ HMMgS } & 270.7 & 6.241 & 0.976 \\
\hline & \multicolumn{3}{|c|}{ Dubinin-Radushkevich model } \\
\hline Sample & $\begin{array}{l}K \\
\left(\mathrm{~mol}^{2} \mathrm{~kJ}^{-2}\right)\end{array}$ & $Q_{\max }\left(\mathrm{mg} \mathrm{g}^{-1}\right)$ & $R^{2}$ \\
\hline HMMgS & -0.111 & 528 & 0.7004 \\
\hline
\end{tabular}

the self-aggregation of MB molecules. ${ }^{47,48}$ Dye molecules like MB tend to form dimers through dimerization in the aqueous solution when the concentration of dye is quite low. Further aggregation could take place between dimers. Hence, parts of the surfaces of HMMgS may adsorb MB aggregates rather than single MB molecules, resulting in more than one layer adsorption of MB. Consequently, HMMgS demonstrates an adsorption capacity higher than the theoretical value.

Additional, if the adsorption capacity is calculated on the basis of surface area, more information could be revealed. MOSF and HMMgS have the similar macroporous structure but different surface area, while the MB adsorption capacity of HMMgS $\left(1.06 \mathrm{mg} \mathrm{m}^{-2}\right)$ is about 4 times higher than that of $\operatorname{MOSF}\left(0.26 \mathrm{mg} \mathrm{m}^{-2}\right)$, suggesting that the enhanced adsorption performance of $\mathrm{HMMgS}$ is mainly attributed to its larger interfaces. Compared with other previously reported magnesium silicate based adsorbents with similar surface area but different nanostructure (Table S1 $\dagger$ ), like magnesium silicate nanotube ${ }^{22,28}$ or hollow sphere, ${ }^{10}$ HMMgS also exhibits the highest $\mathrm{MB}$ adsorption capacity per unit surface area, indicating that the distinctive hierarchical macroporous-mesoporous nanostructure of HMMgS leads to the improvement of the MB adsorption performance. For an adsorbent with high removal efficiency, large surface area is indeed of great importance. However, under the similar surface area, the percentage of the surface area that can be utilized in the adsorption process is the crucial factor for the high adsorption efficiency. The adsorption of positively charged MB molecules occurs on the surface of magnesium silicate with negative charges through strong electrostatic force. In the cases of magnesium silicate nanotube or hollow sphere, MB molecules can access the outer surface of nanotube or hollow sphere easily, and thus most of the outer surface can be used to adsorb MB. In contrast, the interior surfaces of nanotube or hollow sphere with the thickness of 
correlation coefficient of pseudo-second-order kinetic model is much higher than that of pseudo-first-order kinetic model, suggesting the pseudo-second-order kinetic model is suitable to describe the adsorption kinetics of MB by HMMgS. The rate constant related to the adsorption rate is calculated to be $0.003 \mathrm{~g} \mathrm{mg}^{-1} \mathrm{~min}^{-1}$, further indicating the fast MB adsorption rate of HMMgS due to its hierarchical macroporous-mesoporous nanostructure. In addition, calculated $q_{\text {eq }}$ value according to the pseudo-second-order kinetic model is $200 \mathrm{mg} \mathrm{g}^{-1}$, matching well with the experimental data of $199.3 \mathrm{mg} \mathrm{g}^{-1}$. This result further indicates the validity of describing the MB adsorption process by HMMgS using the pseudo-second-order kinetic model.

Pseudo-second-order kinetic model cannot reveal the diffusion mechanism during the whole adsorption process. To solve this issue, the Weber-Morris intra-particle diffusion model was used to analyse the kinetic data. The plot of $q_{t}$ versus $t^{1 / 2}$ is demonstrated in Fig. 4d. Three separate portions can be seen, and data points in each potion can be related by a straight line. The multilinear characteristic suggests three steps are involved in the adsorption process. The rate constant of each step can be calculated from the slopes of straight lines as listed in Table 2 . The first linear portion represents the diffusion of MB molecules from the liquid to the macroporous surfaces (also called film diffusion $),{ }^{30}$ in which the rapid adsorption of $\mathrm{MB}$ can be clearly seen with the first $10 \mathrm{~min}$ as identified by the rate constant of $6.622 \mathrm{mg} \mathrm{g}^{-1} \mathrm{~min}^{-0.5}$. In this step, most of the active sites on the macroporous surface are readily available for MB molecules, and hence $81.7 \%$ of $\mathrm{MB}$ can be adsorbed in this section. When the macroporous surface reaches saturation, MB molecules tends to diffuse into the less accessible pores and are adsorbed by the interior surface of the pores, which are mesopores formed by the stacking of nanosheets of magnesium silicate in the current case. The mesopore diffusion (or intraparticle diffusion) is exhibited by the second portion. ${ }^{55}$ The diffusion rate constant of the mesopore diffusion is $1.581 \mathrm{mg} \mathrm{g}^{-1} \min ^{0.5}$, much smaller than that of macropore diffusion. The reduction in the rate constant is due to the decrease of the pore dimension and consequent smaller diffusion path, causing the increased diffusion resistance. ${ }^{56}$ A platform representing the final adsorption equilibrium can be seen in the third portion, where MB molecules move very slowly from mesopores to micropores. The rate constant of the third portion is equal to 0 , indicating few micropores are involved in HMMgS. The diffusion processes of MB molecules in the first and second parts have the similar length, suggesting both film diffusion and interparticle diffusion are rate-controlling steps.

In the practical application, wastewater containing dyes may in various $\mathrm{pH}$ conditions and ionic strength. The surface charge of HMMgS may be affected by the $\mathrm{pH}$ of the $\mathrm{MB}$ solution, therefore it is necessary to investigate the effect of $\mathrm{pH}$ on the adsorption performance of HMMgS considering its application in the practical wastewater. The initial MB concentration was set to $600 \mathrm{mg} \mathrm{L}^{-1}$ and the adsorption time was $24 \mathrm{~h}$. The $\mathrm{pH}$ value was tuned in the range of 3-8. As shown in Fig. 5a, the adsorption capacities of $\mathrm{MB}$ first grow from $408 \mathrm{mg} \mathrm{g}^{-1}$ to $582 \mathrm{mg} \mathrm{g}^{-1}$ in the $\mathrm{pH}$ range of 3-5, and then keep almost constant in $\mathrm{pH}$ 5-8. Apparently, compared with HMMgS in
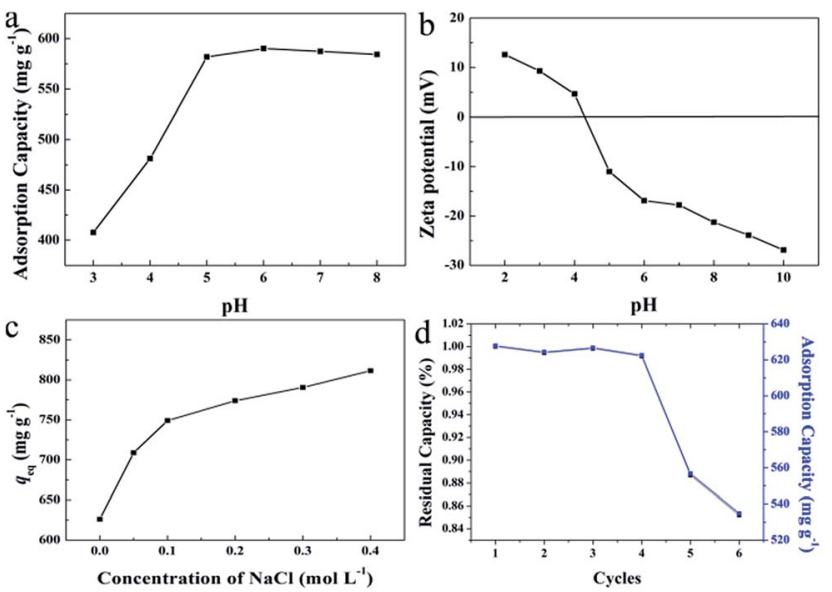

Fig. 5 (a) MB uptake and (b) zeta potential of $\mathrm{HMMgS}$ as a function of $\mathrm{pH}$; (c) the effect of ionic strength on the adsorption capacity of MB; (d) the reusability of $\mathrm{HMMgS}$ after calcination at $450{ }^{\circ} \mathrm{C}$. Initial concentration of MB: $600 \mathrm{mg} \mathrm{L}^{-1}$, the dosage of HMMgS: $0.2 \mathrm{~g} / 40 \mathrm{~mL}$.

strong acid condition, HMMgS in weak acidic to weak basic solution demonstrates much higher MB adsorption capacity. To explain the influence of $\mathrm{pH}$ on the $\mathrm{MB}$ uptake by $\mathrm{HMMgS}$, the zeta-potentials of $\mathrm{HMMgS}$ and $\mathrm{MB}$ solution at different $\mathrm{pH}$ values were measured. The points of zero charge $\left(\mathrm{pH}_{\mathrm{pzc}}\right)$ of HMMgS and MB are measured to be $\sim 4.3$ and $\sim 7.0$ as exhibited in Fig. $5 \mathrm{~b}$ and $\mathrm{S} 2 . \dagger$ At $\mathrm{pH}$ lower than $\mathrm{pH}_{\mathrm{pzc}}$, the active sites on the surface of HMMgS could be occupied by protons, and $\mathrm{MB}$ molecules exist as cations in the solution. Therefore, electrostatic repulsions would exist between HMMgS and MB molecules, causing the low adsorption capacities. When $\mathrm{pH}$ increases to the value higher than $\mathrm{pH}_{\mathrm{pzc}}$ of $\mathrm{HMMgS}$, the surface of HMMgS changes from positive to negative. In the $\mathrm{pH}$ of 3-7, MB molecules have positive charges, and electrostatic interaction between HMMgS and MB would occur, leading to the enhancement of $\mathrm{MB}$ adsorption capacities in the $\mathrm{pH}$ range 3-5. With the adsorption of $\mathrm{MB}$, the active sites on the surface of $\mathrm{HMMgS}$ become saturated, therefore HMMgS in $\mathrm{pH}$ range of 5-7 cannot adsorb more $\mathrm{MB}$, representing by the plateau in Fig. 5a. When $\mathrm{pH}$ is higher than 7, MB molecules show the negatively charged property, and electrostatic repulsions would occur between HMMgS and MB molecules. However, the deterioration in the $\mathrm{MB}$ adsorption capacities cannot be observed when $\mathrm{pH}$ is higher than 7, which can be explained by the dimerization of $\mathrm{MB}$ molecules in the solution. Due to the formation of MB dimers, HMMgS adsorbs MB aggregates rather than single $\mathrm{MB}$ molecules, leading to the almost constant adsorption capacities in the $\mathrm{pH}$ range higher than 7 .

To evaluate the influence of ionic strength on MB adsorption capacity, $\mathrm{NaCl}$ was used to adjust the ionic strength of $\mathrm{MB}$ solution (600 $\mathrm{mg} \mathrm{L}^{-1}$ ) without tuning the $\mathrm{pH}$. Ideally, by increasing the ionic strength, the uptake capacity of MB can be enhanced or decreased when the electrostatic forces between the surface of $\mathrm{HMMgS}$ and $\mathrm{MB}$ ions are repulsive or attractive ${ }^{49,57,58}$ respectively. However, a completely conflicting result was observed from the experiment data. As demonstrated in Fig. 5c, an increase in MB adsorption capacity from 626 to 
$801 \mathrm{mg} \mathrm{g}^{-1}$ can be clearly seen when the concentration of $\mathrm{NaCl}$ ranges from 0 to $0.4 \mathrm{~mol} \mathrm{~L}^{-1}$, suggesting the uptake capacity of positively charged MB by negatively charged HMMgS can be enlarged by increasing the ionic strength. Such conflict can be explained by the dimerization of MB molecules in the solution. As mentioned before, dye molecules tend to form dimers in the solution, and further aggregation could take place between dimers through van der Waals forces, ion dipole and dipoledipole interactions, and dispersion forces originating from delocalised electrons. ${ }^{57}$ The presence of $\mathrm{NaCl}$ can intensify the dimerization of dye molecules. Therefore, with the increase of $\mathrm{NaCl}$ concentration, the aggregation of MB molecules becomes more intensive, and the surfaces of HMMgS adsorb MB aggregates rather than single MB molecules, leading to the growth of MB uptake capacity after the addition of NaCl.

The regeneration ability is also an essential criterion for a good adsorbent in the practical application. Considering the operation difficulty in practical, the adsorbent must be easily separated from the aqueous suspension after adsorption. Previous studies on magnesium silicate based adsorbents with hollow or core/yolk-shell structures normally use centrifugation or magnetic separation to achieve the fully separation of adsorbents from the solution, ${ }^{16,19,25}$ which may cause the additional cost and difficulty in operation. In contrast, $\mathrm{HMMgS}$ has an aggregated spherical morphology with large particle size, therefore, after adsorption it can be easily deposited on the bottom of the vessel by gravitational sedimentation within 30 min (Scheme 1c). Such result indicates HMMgS has the potential to be reused in wastewater treatment. In addition, whether the high adsorption capacity can still be maintained after several cycles of regeneration also determines the regeneration ability of HMMgS. To test the reusability of HMMgS, HMMgS after MB adsorption was regenerated by the calcination at $450{ }^{\circ} \mathrm{C}$ in air for $2 \mathrm{~h}$ to remove adsorbed $\mathrm{MB}$ molecules. Freshly prepared HMMgS shows the MB adsorption uptake of $627 \mathrm{mg} \mathrm{g}^{-1}$. After 4 cycles, HMMgS still keeps the excellent adsorption capacity and no more than $1 \%$ deterioration in the adsorption capacity can be found. Although a decrease in the capacity can be clearly seen from the fifth to the sixth cycles, $85 \%$ of the initial capacity is still retained, indicating the extraordinary cyclic stability of HMMgS. Consequently, HMMgS is an excellent adsorbent for the removal of dyes like MB due to its hierarchical macroporous-mesoporous nanostructure, high MB uptake, good cyclic stability and easy separation.

Since as-prepared HMMgS has the negatively charged surfaces, it can also be utilized as the efficient adsorbent for the removal of heavy-metal ions. Here, we chose lead ions as an example. Fig. S3a and $\mathrm{b} \uparrow$ illustrate the adsorption data of $\mathrm{Pb}^{2+}$ by HMMgS and MOSF, which can be well fitted to Langmuir and Freundlich isotherms, respectively. The maximum adsorption capacity of $\mathrm{HMMgS}$ for $\mathrm{Pb}^{2+}$ is calculated to be $253 \mathrm{mg} \mathrm{g}^{-1}$, while pristine MOSF demonstrates the $\mathrm{Pb}^{2+}$ adsorption capacity of only $52 \mathrm{mg} \mathrm{g}^{-1}$. According to the results of kinetic experiments (Fig. S3c and Table S3†), the adsorption equilibrium of $\mathrm{Pb}^{2+}$ can be reached within $16 \mathrm{~h}$, while other adsorbents like carbon nanotube sheets need more than $70 \mathrm{~h}$ to reach equilibrium (Table S2 $\dagger$ ), demonstrating the fast adsorption rate of HMMgS.
The corresponding rate constant calculated by the pseudosecond-order kinetic model is $0.0027 \mathrm{~g} \mathrm{mg}^{-1} \mathrm{~h}^{-1}$, demonstrating HMMgS is also an efficient adsorption for the removal of heavy metal ions.

To demonstrate HMMgS is an excellent adsorbent to remove various kinds of dyes and heavy metal ions from aqueous solution, anionic dyes methyl orange (MO) and acid blue 83 (AB83), cationic dye methyl blue (MLB), and heavy metal ion $\mathrm{Cr}(\mathrm{vI})$ were also selected as the contaminants to evaluate the adsorption performance of HMMgS. After adsorption for $24 \mathrm{~h}$, $1 \mathrm{~g}$ of HMMgS can remove $282 \mathrm{mg}$ MO, $304 \mathrm{mg} \mathrm{AB83,} 1152 \mathrm{mg}$ MLB and $116 \mathrm{mg} \mathrm{Cr}(\mathrm{vI})$ (Fig. S4 †), showing the wide applicability of HMMgS as the adsorbent for the removal of dyes and heavy metal ions.

\section{Conclusions}

In summary, we have successfully fabricated hierarchical macroporous-mesoporous magnesium silicate as the highly efficient adsorbent for $\mathrm{MB}$ and $\mathrm{Pb}^{2+}$ adsorption by the facile hydrothermal strategy using MOSF as the sacrificial template. The excellent adsorption performance of $\mathrm{HMMgS}$ can be contributed to the large surface area, high pore volume, and the hierarchical nanostructure composed of macropores and mesopores. The large surface area and high pore volume of HMMgS lead to the increase of active sites on the interfaces, while the existence of macropores and mesopores facilitate the mass transportation and diffusion of contaminants to the interfaces, resulting in the enhancement of the usage efficiency of active sites and consequent excellent adsorption capacities (602 $\mathrm{mg} \mathrm{g}^{-1}$ for MB and $253 \mathrm{mg} \mathrm{g}^{-1}$ for $\mathrm{Pb}^{2+}$ ). Besides, $\mathrm{HMMgS}$ also shows good recyclability as proved by its easy separation and regeneration process, demonstrating its potential in practical wastewater treatment. The facile hydrothermal strategy using MOSF as the sacrificial template might be extended to fabricate other metal silicates with similar hierarchical porous nanostructure, which may have great potential in applications such as catalysis and lithium ion battery because of the enhanced mass transportation efficiency.

\section{Conflicts of interest}

There are no conflicts to declare.

\section{Acknowledgements}

This work was financially supported by the National Natural Science Foundation of China (Grant No. 51502181). The authors thank the support from Experimental Testing Centre, College of Chemistry, Sichuan University and Analytical \& Testing Centre of Sichuan University for sample analysis.

\section{Notes and references}

1 C. J. Vörösmarty, P. B. Mcintyre, M. O. Gessner, D. Dudgeon, A. Prusevich, P. Green, S. Glidden, S. E. Bunn, C. A. Sullivan and C. R. Liermann, Nature, 2010, 467, 555. 
2 E. Forgacs, T. Cserháti and G. Oros, Environ. Int., 2004, 30, 953-971.

3 M. M. Ayad and A. A. Elnasr, J. Phys. Chem. C, 2010, 114, 2177-2187.

4 P. Cañizares, F. Martínez, C. Jiménez, J. Lobato and M. A. Rodrigo, Environ. Sci. Technol., 2006, 40, 6418.

5 L. G. Devi, S. G. Kumar, K. M. Reddy and C. Munikrishnappa, J. Hazard. Mater., 2009, 164, 459-467.

6 W. Kang, C. Spanjers, R. Rioux and J. Hoefelmeyer, J. Mater. Chem. A, 2013, 1, 7717-7728.

7 C. C. Striemer, T. R. Gaborski, J. L. Mcgrath and P. M. Fauchet, Nature, 2007, 445, 749-753.

8 J. Qu, C. Y. Cao, Y. L. Hong, C. Q. Chen, P. P. Zhu, W. G. Song and Z. Y. Wu, J. Mater. Chem., 2012, 22, 3562-3567.

9 L. Zhang and M. Fang, Nano Today, 2010, 5, 128-142.

10 Y. Q. Wang, G. Z. Wang, H. Q. Wang, C. H. Liang, W. P. Cai and L. D. Zhang, Chem.-Eur. J., 2010, 16, 3497-3503.

11 R. X. Jin, Y. Yang, Y. F. Li, X. D. Yu, Y. Xing, S. Y. Song and Z. Shi, ChemPlusChem, 2015, 80, 544-548.

12 J. Dai, J. Sun, A. Xie, J. He, C. Li and Y. Yan, $R S C$ Adv., 2016, 6, 3446-3457.

13 M. Akgül and A. Karabakan, Microporous Mesoporous Mater., 2011, 145, 157-164.

14 K. Y. Kumar, H. B. Muralidhara, Y. A. Nayaka, J. Balasubramanyam and H. Hanumanthappa, Powder Technol., 2013, 246, 125-136.

15 R. Jin, Y. Yang, Y. Xing, L. Chen, S. Song and R. Jin, ACS Nano, 2014, 8, 3664.

16 R. Y. Huang, M. J. Wu, T. Zhang, D. Q. Li, P. G. Tang and Y. J. Feng, ACS Sustainable Chem. Eng., 2017, 5, 2774-2780.

17 A. Corma, ChemInform, 1997, 28, 2373.

18 S. Zhang, W. Xu, M. Zeng, J. Li, J. Li, J. Xu and X. Wang, J. Mater. Chem. A, 2013, 1, 11691-11697.

19 B. F. Zou, K. Chen, Y. Q. Wang, C. Y. Niu and S. M. Zhou, $R S C$ Adv., 2015, 5, 22973-22979.

20 Y. C. Yu, Z. J. Hu, Z. Y. Chen, J. X. Yang, H. W. Gao and Z. W. Chen, RSC Adv., 2016, 6, 97523-97531.

21 Z. Wu and D. Zhao, Chem. Commun., 2011, 47, 3332.

22 J. Qu, W. Li, C. Y. Cao, X. J. Yin, L. Zhao, J. Bai, Z. Qin and W. G. Song, J. Mater. Chem., 2012, 22, 17222-17226.

23 C. X. Gui, Q. Q. Wang, S. M. Hao, J. Qu, P. P. Huang, C. Y. Cao, W. G. Song and Z. Z. Yu, ACS Appl. Mater. Interfaces, 2014, 6, 14653-14659.

24 J. Zheng, B. H. Wu, Z. Y. Jiang, Q. Kuang, X. L. Fang, Z. X. Xie, R. B. Huang and L. S. Zheng, Chem.-Asian J., 2010, 5, 14391444.

25 C. Y. Cao, F. Wei, J. Qu and W. G. Song, Chem.-Eur. J., 2013, 19, 1558-1562.

26 Y. H. Kotp, Sep. Sci. Technol., 2017, 52, 657-670.

27 Q. Li, J. Zhang, Q. Lu, J. Lu, J. Li, C. Dong and Q. Zhu, Mater. Lett., 2016, 170, 167-170.

28 Y. Tian, G. Cui, Y. Liu, H. Li, Z. Sun and S. Yan, Appl. Surf. Sci., 2016, 387, 631-641.

29 J. Zheng, C. Cheng, R. W. Yan, W. J. Fang, C. Chen, H. X. Huai and C. C. Wang, J. Alloys Compd., 2014, 596, 5-9.
30 S. J. Allen, G. Mckay and K. Y. Khader, Environ. Pollut., 1989, 56, 39-50.

31 J. C. Y. Ng, Sep. Purif. Rev., 2000, 29, 189-232.

32 H. Wang, X. Zhou, M. Yu, Y. Wang, L. Han, J. Zhang, P. Yuan, G. Auchterlonie, J. Zou and C. Yu, J. Am. Chem. Soc., 2006, 128, 15992-15993.

33 P. Yuan, X. Zhou, H. Wang, N. Liu, Y. Hu, G. J. Auchterlonie, J. Drennan, X. Yao, G. Q. Lu and J. Zou, Small, 2009, 5, 377382.

34 H. N. Wang, X. F. Zhou, M. H. Yu, Y. H. Wang, L. Han, J. Zhang, P. Yuan, G. Auchterlonie, J. Zou and C. Z. Yu, J. Am. Chem. Soc., 2006, 128, 15992-15993.

35 K. R. Hall, L. C. Eagleton, A. Acrivos and T. Vermeulen, Ind. Eng. Chem. Fundam., 1966, 5, 587-594.

36 Y. S. Ho and G. Mckay, Chem. Eng. J., 1998, 70, 115-124.

37 G. H. Sonawane and V. S. Shrivastava, Desalin. Water Treat., 2011, 29, 29-38.

38 W. J. Weber and J. C. Morris, J. Sanit. Eng. Div., 1963, 89, 3160.

39 E. M. E. Kristensen, F. Nederberg, H. Rensmo, T. Bowden, J. Hilborn and H. Siegbahn, Langmuir, 2006, 22, 9651-9657. 40 H. Zhu, B. Lee, D. Sheng and S. H. Overbury, Langmuir, 2003, 19, 3974-3980.

41 S. Jambhrunkar, M. Yu, J. Yang, J. Zhang, A. Shrotri, L. Endomunoz, J. Moreau, G. Lu and C. Yu, J. Am. Chem. Soc., 2013, 135, 8444-8447.

42 T. W. Sun, Y. J. Zhu, C. Qi, F. Chen, Y. Y. Jiang, Y. G. Zhang, J. Wu and C. Wu, J. Mater. Chem. B, 2016, 4, 3257-3268.

43 S. S. Chao, Y. Takagi, G. Lucovsky, P. Pai, R. C. Custer, J. E. Tyler and J. E. Keem, Appl. Surf. Sci., 1986, 26, 575-583. 44 K. S. W. Sing, Pure Appl. Chem., 1985, 57, 603-619.

45 W. W. Lukens, P. Schmidtwinkel, D. Zhao, J. Feng and G. D. Stucky, Langmuir, 1999, 15, 5403-5409.

46 D. Lin and B. Xing, Environ. Sci. Technol., 2008, 42, 7254.

47 G. M. Walker and L. R. Weatherley, Chem. Eng. J., 2001, 83, 201-206.

48 E. Coates, Color. Technol., 1969, 85, 355-368.

49 J. Ma, F. Yu, L. Zhou, L. Jin, M. Yang, J. Luan, Y. Tang, H. Fan, Z. Yuan and J. Chen, ACS Appl. Mater. Interfaces, 2012, 4, 5749.

50 B. M. Jovanović, V. L. Vukašinović-Pešić and L. V. Rajaković, Water Environ. Res., 2011, 83, 498-506.

51 J. P. Hobson, J. Phys. Chem., 1969, 73, 2720-2727.

52 M. S. Onyango, Y. Kojima, O. Aoyi, E. C. Bernardo and

H. Matsuda, J. Colloid Interface Sci., 2004, 279, 341.

53 W. T. Elwell, Anal. Chim. Acta, 1971, 54, 378.

54 M. Mahramanlioglu, I. Kizilcikli and I. O. Bicer, J. Fluorine Chem., 2002, 115, 41-47.

55 S. Rengaraj, Y. Kim, C. K. Joo and J. Yi, J. Colloid Interface Sci., 2004, 273, 14-21.

56 C. S. Cheng, J. Deng, B. Lei, A. He, X. Zhang, L. Ma, S. Li and C. Zhao, J. Hazard. Mater., 2013, 263, 467-478.

57 G. Alberghina, R. Bianchini, M. Fichera and S. Fisichella, Dyes Pigm., 2000, 46, 129-137.

58 Y. S. Al-Degs, M. I. El-Barghouthi, A. H. El-Sheikh and G. M. Walker, Dyes Pigm., 2008, 77, 16-23. 